\title{
Intermittent parathyroid hormone (1-34) application regulates cAMP-response element binding protein activity to promote the proliferation and osteogenic differentiation of bone mesenchymal stromal cells, via the cAMP/PKA signaling pathway
}

\author{
BAILING CHEN ${ }^{1 *}$, TAO LIN $^{1 *}$, XIAOXI YANG ${ }^{2},{\text { YIQIANG } \text { LI }^{3}, \text { DENGHUI XIE }^{4} \text { and HAOWEN CUI }}^{1}$ \\ ${ }^{1}$ Department of Spine Surgery, The First Affiliated Hospital of Sun Yat-sen University, Guangzhou, Guangdong 510080; \\ ${ }^{2}$ Department of Spine Surgery, Chinese PLA General Hospital (301 Hospital), Beijing 100853; ${ }^{3}$ Department of Orthopedics, \\ Guangzhou Women and Children's Medical Center, Guangzhou, Guangdong 510623; ${ }^{4}$ Department of Spine Surgery, \\ The Third Affiliated Hospital of Southern Medical University, Guangzhou, Guangdong 510630, P.R. China
}

Received January 24, 2015; Accepted March 1, 2016

DOI: $10.3892 / \mathrm{etm} .2016 .3177$

\begin{abstract}
The potential effects of intermittent parathyroid hormone (1-34) [PTH (1-34)] administration on bone formation have previously been investigated. A number of studies have suggested that the cyclic adenosine monophosphate/protein kinase A (cAMP/PKA) pathway is associated with $\mathrm{PTH}$-induced osteogenic differentiation. However, the precise signaling pathways and molecular mechanism by which PTH (1-34) induces the osteogenic differentiation of bone mesenchymal stromal cells (BMSCs) remain elusive. The purpose of the present study was to investigate the mechanism underlying the effect of intermittent PTH (1-34) application on the proliferation and osteogenic differentiation of BMSCs. BMSCs were randomly divided into four groups, as follows: Osteogenic medium (control group); osteogenic medium and intermittent PTH (1-34); osteogenic medium and intermittent PTH (1-34) plus the adenylyl cyclase activator forskolin; and osteogenic medium and intermittent PTH (1-34) plus the PKA inhibitor $\mathrm{H}-89$. A cell proliferation assay revealed that PTH (1-34) stimulates BMSC proliferation via the cAMP/PKA pathway. Furthermore, reverse transcription-quantitative polymerase chain reaction, alkaline phosphatase activity testing and cell examination using Alizarin Red S staining
\end{abstract}

Correspondence to: Professor Bailing Chen, Department of Spine Surgery, The First Affiliated Hospital of Sun Yat-sen University, 58 Zhongshan 2nd Road, Guangzhou, Guangdong 510080, P.R. China

E-mail: chenbl2012@163.com

*Contributed equally

Key words: bone mesenchymal stromal cells, osteogenic differentiation, parathyroid hormone (1-34), cyclic adenosine monophosphate/protein kinase A pathway demonstrated that PTH (1-34) administration promotes osteogenic differentiation and mineralization, mediated by the cAMP/PKA pathway. Crucially, the results of western blot analyses suggested that PTH (1-34) treatment and, to a greater degree, PTH (1-34) plus forskolin treatment caused an increase in phosphorylated cAMP response element binding protein (p-CREB) expression, but the effect of PTH on p-CREB expression was blocked by $\mathrm{H}-89$. In conclusion, the current study demonstrated that intermittent PTH (1-34) administration regulates downstream proteins, particularly p-CREB, in the cAMP/PKA signaling pathway, to enhance the proliferation, osteogenic differentiation and mineralization of BMSCs.

\section{Introduction}

Parathyroid hormone (1-34) [PTH (1-34)] is currently the only anabolic agent approved by the Food and Drug Administration (FDA) for the treatment of osteoporosis in the USA (1). It is established that intermittent PTH (1-34) administration increases mass, strength and mineral density of bone, and improves bone microarchitecture and fracture healing (2-4). Furthermore, previous in vitro studies have indicated that intermittent delivery of PTH (1-34) enhances the proliferation and differentiation of osteoprogenitor cells in bone marrow, increases osteoblast activity and inhibits osteoblast apoptosis (5-7). However, the precise molecular mechanism underlying the effect of PTH (1-34) during the osteogenic differentiation of bone mesenchymal stromal cells (BMSCs) remains elusive.

A number of previous studies have reported that PTH affects osteoblastic cells through activation of cyclic adenosine monophosphate/protein kinase A (cAMP/PKA) $(8,9)$, Wnt/ $\beta$-catenin (10-12) and mitogen-activated protein kinase signaling pathways $(13,14)$; however, the focus of previous studies has particularly been on cAMP signaling. Several previous studies have evaluated the role of PKA pathway in osteogenic differentiation of hMSCs, and reported that pretreatment of human MSCs with a cAMP analog or 
forskolin enhanced bone formation (15-17). cAMP is a pivotal intracellular signaling molecule, the main function of which is to activate the cAMP-dependent PKA (18). Adenylate cyclase is activated through dissociated G-proteins, causing the conversion of adenosine triphosphate into cAMP (17). Subsequently, cAMP activates PKA, the cAMP-responsive element binding (CREB) protein is phosphorylated, and this translocates into the nucleus where it activates transcription of target genes (17).

PTH signaling is mediated by a $\mathrm{G}$ protein-coupled receptor, known as parathyroid receptor 1 (PTHR1) (19). Ligand binding to PTHR1 stimulates the activation of adenylate cyclase mediated by the $G$ protein subunit $G_{a s}$, thereby stimulating cAMP production and the subsequent activation of PKA. Wang et al (8) treated the rat osteoblast-like cell line UMR 106 with PTH (1-34), revealing that PTH stimulates the expression of the transcription factors runt related transcription factor 2 (RUNX2) and osterix in vitro, and that induction of RUNX2 mRNA expression is mediated through the activation of the cAMP/PKA pathway. In another study, Nakao et al (9) evaluated cAMP and bone morphogenetic protein (BMP) levels in MC3T3-E1 cells following the addition of PTH, and found that PTH enhanced BMP activity by increasing cAMP accumulation in MC3T3-E1 cells.

Although a number of previous studies have demonstrated that the effects of PTH (1-34) are associated with the cAMP/PKA pathway, it is unclear how intermittent administration of PTH (1-34) regulates osteogenic differentiation of BMSCs through the cAMP/PKA pathway. The present study therefore aimed to investigate the molecular mechanism of intermittent PTH (1-34) application in the regulation of the proliferation and osteogenic differentiation of BMSCs by the cAMP/PKA pathway.

\section{Materials and methods}

Isolation and culture of rat BMSCs. A total of 20 male Sprague-Dawley rats (age, 5 weeks; weight, 100-120 g) were purchased from the Experimental Animal Center of Sun Yat-Sen University (Guangzhou, China) for use in the present study. The rats were acclimated to the housing conditions for 7 days, during which the rats were maintained under a 12-h light/dark cycle at $22^{\circ} \mathrm{C}$ with ad libitum access to food and water. The current study was approved by the Animal Care Committee of Sun Yat-Sen University and was in compliance with guiding principles for the use of laboratory animals (20). Following sacrifice of the rats by overdose with $10 \%$ chloral hydrate (intraperitoneal injection; Guangzhou Chemical Reagent Factory, Guangzhou, China), the femora and tibiae of the rats were removed. The ends of each bone were dissected and the BMSCs were harvested by flushing out the bone marrow with $5 \mathrm{ml}$ Dulbecco's modified Eagle's medium (DMEM; Gibco; Thermo Fisher Scientific, Inc., Waltham, MA, USA) twice using a syringe. The BMSCs were centrifuged at $1,000 \times \mathrm{g}$ for $5 \mathrm{~min}$, resuspended in DMEM containing $10 \%$ fetal bovine serum (FBS; Gibco; Thermo Fisher Scientific, Inc.) and $1 \%$ penicillin/streptomycin (Gibco; Thermo Fisher Scientific, Inc.) and incubated at $37^{\circ} \mathrm{C}$ with $5 \% \mathrm{CO}_{2}$. The culture medium was changed every 2 days. Upon reaching $80-90 \%$ confluence, the cells were subcultured. BMSCs at passage 3 were used in all experiments.
PTH (1-34), forskolin and $H$-89 administration. For all experiments, BMSCs were randomly divided into four groups, as follows: No PTH treatment (control); PTH (1-34) treatment (PTH; ProSpec-Tany TecnoGene Ltd., Rehovot, Israel); PTH (1-34) plus treatment with forskolin (FSK; Beyotime Institute of Biotechnology, Haimen, China), an adenylyl cyclase activator; and PTH (1-34) plus treatment with a PKA inhibitor (H-89; Beyotime Institute of Biotechnology).

For the control group, $24 \mathrm{~h}$ after culture, the medium was changed to fresh DMEM medium for $6 \mathrm{~h}$, and then the cells were cultured in PTH-free osteogenic medium containing $10 \mathrm{nM}$ dexamethasone, $10 \mathrm{mM} \beta$-glycerophosphate, and $50 \mu \mathrm{g} / \mathrm{ml}$ ascorbic acid, (all Sigma-Aldrich, St. Louis, MO, USA) for the remaining $42 \mathrm{~h}$ of each 48 -h cycle. In the PTH group, BMSCs were exposed to $10 \mathrm{nM}$ PTH (1-34) (7) in DMEM medium for the first $6 \mathrm{~h}$ of each 48-h cycle and then washed with phosphate-buffered saline (PBS) and cultured in osteogenic medium for the subsequent $42 \mathrm{~h}$. Similarly, BMSCs in the FSK and H-89 groups were treated for $6 \mathrm{~h}$ with $10 \mathrm{nM}$ PTH (1-34) plus $10 \mathrm{nM}$ forskolin or $10 \mu \mathrm{M} \mathrm{H}-89$ (21), respectively, before being washed with $\mathrm{PBS}$ and cultured in osteogenic medium for $42 \mathrm{~h}$. In all groups, the medium was changed every $48 \mathrm{~h}$, and all groups were cultured for 14 days.

Cell proliferation assay. BMSCs were cultured in 96-well plates at a density of $1 \times 10^{3}$ cells/well, and treated as described above. Cell proliferation was assessed using a Cell Counting kit-8 (CCK-8; Dojindo Molecular Technologies, Inc., Kumamoto, Japan) at 3,7,10 and 14 days. For this, the culture medium in all groups was removed and changed to fresh culture medium. Subsequently, CCK- 8 solution was added $(10 \mu 1$ per well) and incubated for $2 \mathrm{~h}$ at $37^{\circ} \mathrm{C}$ with $5 \% \mathrm{CO}_{2}$. Finally, cell proliferation was assessed on the basis of optical density values, measured at $450 \mathrm{~nm}$ using a Sunrise microplate reader (Tecan Trading AG, Männedorf, Switzerland). Five replicates of each sample at each time point were measured.

Alkaline phosphatase (ALP) activity assessment. BMSCs were seeded in 6-well plates at a density of $5 \times 10^{4}$ cells/well in each group and treated as described above. On days 7 and 14, ALP was extracted and detected using a SensoLyte $p$-nitrophenyl phosphate (pNPP) ALP assay kit (AnaSpec, Inc., Fremont, CA, USA) in accordance with the manufacturer's protocol. Cells were initially lysed using radioimmunoprecipitation assay lysis buffer (Beyotime Institute of Biotechnology), containing $10 \mathrm{mM}$ Tris (pH 7.0), $1 \mathrm{mM}$ EDTA, and $0.2 \%$ Triton $\mathrm{X}-100$, and the lysates were frozen at $-80^{\circ} \mathrm{C}$ and then thawed. Cell lysate was centrifuged for $15 \mathrm{~min}\left(10,000 \mathrm{x} \mathrm{g}, 4^{\circ} \mathrm{C}\right)$, after which the supernatant was collected and combined with pNPP. ALP activity was determined at $405 \mathrm{~nm}$ using the Sunrise microplate reader. The values were normalized to total protein content, measured using a NanoDrop 2000 spectrophotometer (NanoDrop; Thermo Fisher Scientific, Inc., Wilmington, DE, USA).

Reverse transcription-quantitative polymerase chain reaction (RT-qPCR). On day 14, total RNA was extracted from the cultured cells in each group using TRIzol (Invitrogen; Thermo Fisher Scientific, Inc.). Subsequent to RNA isolation, the concentration and purity of RNA was assessed using a 
Table I. Gene primers for quantitative reverse transcription-polymerase chain reaction.

\begin{tabular}{|c|c|c|c|c|}
\hline Gene & Primer & Sequence, 5'-3' & Product size, bp & GenBank accession no. \\
\hline RUNX2 & $\begin{array}{l}\text { Forward } \\
\text { Reverse }\end{array}$ & $\begin{array}{l}\text { GGGAACCAAGAAGGCACAGA } \\
\text { GGTGGAATGGATGGATGGGG }\end{array}$ & 171 & NM_001278484.1 \\
\hline Osterix & $\begin{array}{l}\text { Forward } \\
\text { Reverse }\end{array}$ & $\begin{array}{l}\text { AGGAGACGGGACAGCCAA } \\
\text { AGGAAATGAGTGGGGAAAGGG }\end{array}$ & 113 & NM_001037632.1 \\
\hline Collagen I & $\begin{array}{l}\text { Forward } \\
\text { Reverse }\end{array}$ & $\begin{array}{l}\text { GCGGAGGAGGCTATGACTTT } \\
\text { AGGCGAGATGGCTTATTCGT }\end{array}$ & 162 & NM_053356.1 \\
\hline Osteocalcin & $\begin{array}{l}\text { Forward } \\
\text { Reverse }\end{array}$ & $\begin{array}{l}\text { ACCTGTGGAGCAGAAATGGT } \\
\text { GGCTGAAGTTGGTCGTTTGG }\end{array}$ & 187 & NM_001033860.1 \\
\hline Osteopontin & $\begin{array}{l}\text { Forward } \\
\text { Reverse }\end{array}$ & $\begin{array}{l}\text { AGTGGTTTGCTTTTGCCTGT } \\
\text { AACTCGTGGCTCTGATGTTCC }\end{array}$ & 118 & NM_012881.2 \\
\hline$\beta$-actin & $\begin{array}{l}\text { Forward } \\
\text { Reverse }\end{array}$ & $\begin{array}{l}\text { GGAAATCGTGCGTGACATTA } \\
\text { CCACCCGTCCTCCAGTCC }\end{array}$ & 173 & NM_031144.3 \\
\hline
\end{tabular}

RUNX2, runt related transcription factor 2 .

NanoDrop 2000 spectrophotometer. A total of 1,000 ng RNA per group was used for cDNA synthesis using a SYBR-PrimeScript RT-PCR kit (Takara Biotechnology Co., Ltd., Dalian, China). This included $0.5 \mu 1$ PrimeScript RT Enzyme mix I, $0.5 \mathrm{ml}$ oligo dT primer, and $2 \mathrm{ml}$ PrimeScript Buffer to a final volume of $10 \mu \mathrm{l}$. A total of $1 \mu \mathrm{l}$ cDNA was then used for RT-qPCR in a $10-\mu 1$ reaction volume containing $0.3 \mu$ l forward and $0.3 \mu 1$ reverse primers (Table I), $5 \mu \mathrm{l} \mathrm{SYBR}$ Premix Ex Taq (Takara Biotechnology Co., Ltd.) and $3.4 \mu \mathrm{l}$ diethylpyrocarbonate-treated water (Takara Biotechnology Co., Ltd.). qPCR was performed on each cDNA sample in triplicate under the following cycling conditions: Initial denaturation at $95^{\circ} \mathrm{C}$ for $5 \mathrm{~min}$, followed by 40 cycles of denaturation at $94^{\circ} \mathrm{C}$ for $30 \mathrm{sec}$, annealing at $60^{\circ} \mathrm{C}$ for $10 \mathrm{sec}$ and elongation at $72^{\circ} \mathrm{C}$ for $20 \mathrm{sec}$, and a final extension step at $72^{\circ} \mathrm{C}$ for $10 \mathrm{~min}$. Fluorescence data was analyzed using a CFX96 Real-Time PCR Detection system (Bio-Rad Laboratories, Inc., Hercules, CA, USA). Gene expression levels were calculated using the $2^{-\Delta \Delta C q}$ method (22). Data are presented as fold change relative to control samples.

Alizarin Red S staining. To observe the osteogenic differentiation of BMSCs, cells were subjected to Alizarin Red S staining. Cells were seeded in 24 -well plates at a density of $5 \times 10^{3}$ cells/well. Following the 14-day culture, the osteogenic medium was removed from the cells in each group. Cells were then washed twice with ice-cold PBS and fixed with $70 \%$ ethanol for $30 \mathrm{~min}$. Next, cells were stained with $1 \%$ Alizarin Red S solution ( $\mathrm{pH} 4.2$; Sigma-Aldrich) for $15 \mathrm{~min}$ and gently rinsed with $\mathrm{dH}_{2} \mathrm{O}$. The samples were imaged with an Axio Imager Z1 microscope (Zeiss AG, Oberkochen, Germany).

Western blot analysis. Following the 14-day culture, cells in each group were washed twice with ice-cold PBS and lysed in $80 \mu \mathrm{l}$ Mammalian Protein Extraction reagent and protease inhibitor cocktail tablets (both Thermo Fisher Scientific, Inc.). The samples were centrifuged for $15 \mathrm{~min}\left(12,000 \mathrm{x} \mathrm{g}, 4^{\circ} \mathrm{C}\right)$, the supernatant was extracted and total protein concentration was determined using a NanoDrop 2000 spectrophotometer. The samples were denatured by heating for $5 \mathrm{~min}$ at $95^{\circ} \mathrm{C}$ in loading buffer (Beyotime Institute of Biotechnology), containing 0.5 M Tris $\cdot \mathrm{HCl}, 0.5 \mathrm{M}$ DTT, sodium dodecyl sulfate (SDS), bromophenol blue and glycerol. Subsequently, equal volumes of the samples $(20 \mu \mathrm{l})$ were run on $12 \%$ SDS-polyacrylamide gels and transferred to polyvinylidene fluoride membranes. The membranes were blocked for $1 \mathrm{~h}$ with blocking reagent (Tris-buffered saline containing 5\% nonfat milk powder) and incubated overnight at $4^{\circ} \mathrm{C}$ with primary antibodies, as follows: Rabbit anti-phosphorylated CREB (p-CREB) monoclonal antibody (mAb; 1:1,000; cat. no. 9198), rabbit anti-CREB mAb (1:1,000; cat. no. 9197), rabbit anti-RUNX2 mAb (1:1,000; cat. no. 12556), rabbit anti-osterix $m A b(1: 1,000$; cat. no. 13572) and rabbit anti- $\beta$-tubulin mAb (1:1,000; \#15115; all Cell Signaling Technology, Inc., Danvers, MA, USA). The membrane was then incubated with alkaline phosphatase-conjugated goat anti-IgG (1:2,000, cat. no. 7054; Cell Signaling Technology) for $1 \mathrm{~h}$ at room temperature, and the proteins were visualized using a chemiluminescence kit (EMD Millipore, Billerica, MA, USA). Densitometric analysis was performed using Photoshop CS5 (Adobe Systems, Inc., San Jose, CA, USA).

Statistical analysis. All results are expressed as the mean \pm standard deviation. Comparative studies of means were performed by one-way analysis of variance and Fisher's least significant difference test, using SPSS version 13.0 (SPSS, Inc., Chicago, IL, USA) and a statistically significant difference was defined as $\mathrm{P}<0.05$. All experiments were repeated in triplicate.

\section{Results}

Intermittent PTH (1-34) administration regulates proliferation of BMSCs via the cAMP/PKA pathway. As shown in Fig. 1, the cell number, as determined by the optical density using a cell counting kit, gradually increased over time in all four groups. After 3, 7, 10 and 14 days of intermittent PTH (1-34) treatment, an increase in cell number was observed in the PTH group 


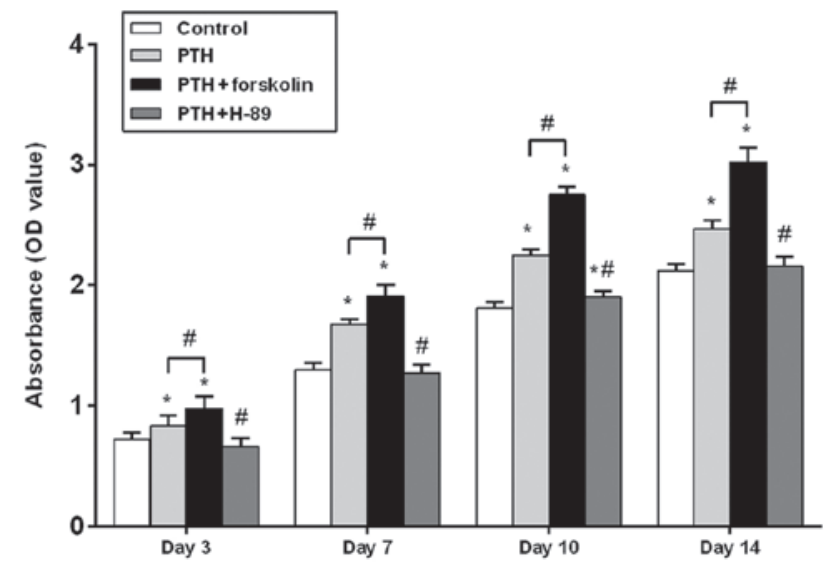

Figure 1. Effect of intermittent PTH (1-34) administration on bone mesenchymal stromal cell proliferation through the cAMP/PKA pathway. Cell number increased over time in all groups, as assessed by OD. PTH (1-34) increased cell number at each time point. Following activation of cAMP/PKA signaling by forskolin, the cell number significantly increased on all days measured. Following treatment with PTH (1-34) and H-89, a cAMP/PKA inhibitor, a significant reduction in cell number was observed at each time point. Data are expressed as mean \pm standard deviation ( $\mathrm{n}=3$ repeats). ${ }^{*} \mathrm{P}<0.05$ vs. control group; ${ }^{\text {P }} \mathrm{P}<0.05$ vs. PTH group. PTH (1-34), parathyroid hormone (1-34); OD, optical density; cAMP/PKA, cyclic adenosine monophosphate/protein kinase A.

compared with the control group, and there was a significant difference between these groups at each time point $(\mathrm{P}<0.05)$. In the PTH + FSK group, a significant increase in cell number was observed on days 3, 7, 10 and 14 when compared with the control group $(\mathrm{P}<0.05)$ and with the $\mathrm{PTH}$ group $(\mathrm{P}<0.05)$. Following treatment of PTH (1-34) plus H-89, cell number was significantly decreased compared with that in the PTH group at each time point $(\mathrm{P}<0.05)$. No significant difference was observed between the $\mathrm{PTH}+\mathrm{H}-89$ group and the control groups on days 3, 7 and 14 ( $P>0.05)$, but on day 10 an increase in cell number was observed in the $\mathrm{TH}+\mathrm{H}-89$ group compared with the control group $(\mathrm{P}<0.05)$.

Assessment of ALP activity under different treatment conditions. At days 7 and 14, the PTH and PTH + FSK groups demonstrated significantly increased ALP activity compared with the control group $(\mathrm{P}<0.05)$, and the PTH + FSK group also had significantly increased ALP activity compared with the PTH group $(\mathrm{P}<0.05)$. However, treatment with $\mathrm{H}-89$ reduced ALP activity compared with that in the PTH group ( $\mathrm{P}<0.05$; Fig. 2).

Intermittent PTH (1-34) administration promotes osteogenic gene expression via the $c A M P / P K A$ signaling pathway. RUNX2, osterix, collagen I, osteocalcin and osteopontin mRNA expression levels in the PTH and PTH + FSK groups significantly increased compared with those in the control group $(\mathrm{P}<0.05)$, but the levels of all genes examined were higher in the PTH + FSK group than in the PTH group $(\mathrm{P}<0.05$; Fig. 3). RUNX2, osterix, collagen I, osteocalcin and osteopontin mRNA expression was significantly downregulated in the PTH + H-89 group compared with the PTH group $(\mathrm{P}<0.05)$, but the mRNA expression levels of these (with the exception of osterix) were higher in the PTH $+\mathrm{H}-89$ group than in the

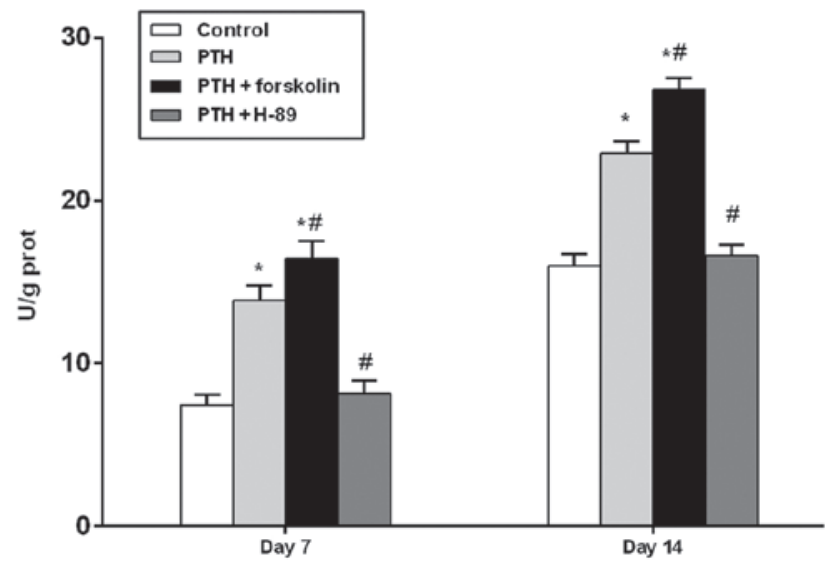

Figure 2. Analysis of ALP activity in each group on days 7 and 14. ALP activity increased with culture time. Intermittent PTH (1-34) treatment significantly upregulated ALP activity. Following activation of cyclic adenosine monophosphate/protein kinase A signaling using forskolin, ALP activity was higher than in the PTH-treated group, and treatment with H-89, an inhibitor of this pathway significantly decreased ALP activity. Data are expressed as mean \pm standard deviation ( $\mathrm{n}=3$ repeats). ${ }^{*} \mathrm{P}<0.05$ vs. control group; ${ }^{~} \mathrm{P}<0.05$ vs. PTH group. PTH (1-34), parathyroid hormone (1-34); ALP, alkaline phosphatase.

control group $(\mathrm{P}<0.05)$. Notably, osterix mRNA expression revealed a moderate but not statistically significant reduction in the PTH $+\mathrm{H}-89$ group compared with the control group.

Effects of intermittent PTH (1-34) application on mineralization of osteoblasts, induced from BMSCs via the cAMP/PKA pathway. As indicated in Fig. 4, the PTH and PTH + FSK groups developed notably increased mineralization compared with the control group (Fig. 4A-C). The mineralization effect of PTH (1-34) plus forskolin was markedly increased from that of PTH (1-34) treatment alone. However, treatment with PTH (1-34) plus H-89 reduced mineralization compared with that in the PTH group (Fig. 4B and D).

Intermittent PTH (1-34) treatment increases osteogenic differentiation of BMSCs, mediated by the $c A M P / P K A$ signaling pathway. Western blotting and a densitometric analysis revealed that p-CREB expression in the PTH group increased significantly compared with that in the control group $(\mathrm{P}<0.05$; Fig. 5A). With the concurrent administration of forskolin, p-CREB expression markedly increased compared with that in the control group $(\mathrm{P}<0.05)$ and the $\mathrm{PTH}$ group $(\mathrm{P}<0.05)$. In the $\mathrm{PTH}+\mathrm{H}-89$ group, $\mathrm{p}-\mathrm{CREB}$ expression was reduced compared with that in the PTH group $(\mathrm{P}<0.05)$, and did not significantly differ from the $\mathrm{p}$-CREB expression in the control group ( $\mathrm{P}>0.05$; Fig. 5A). Conversely, there were no statistical differences in CREB expression between the four groups. (P>0.05; Fig. 5A). RUNX2 and osterix protein levels were significantly increased in the PTH and PTH + FSK groups when compared with the control group $(\mathrm{P}<0.05)$, and the FSK group also exhibited increased expression of these proteins compared with the PTH group $(\mathrm{P}<0.05)$. Significant reductions in RUNX2 and osterix protein expression levels were observed in the PTH $+\mathrm{H}-89$ group compared with the PTH group $(\mathrm{P}<0.05)$, but a difference was not observed compared with the control group (Fig. 5B). 
A

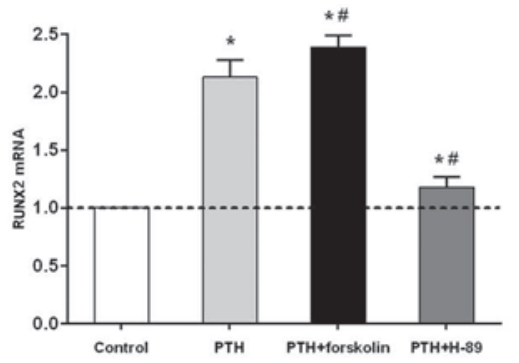

B

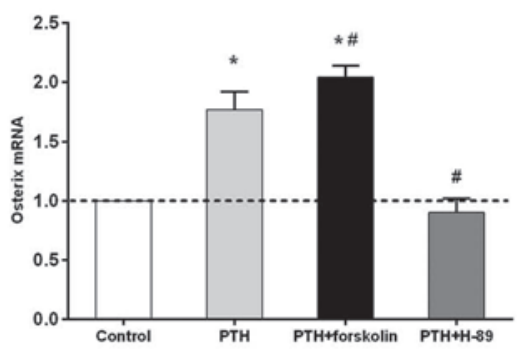

C

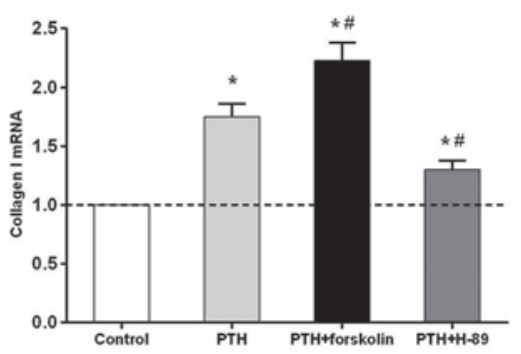

D

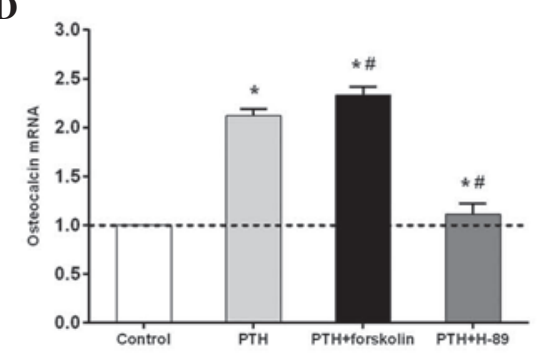

E

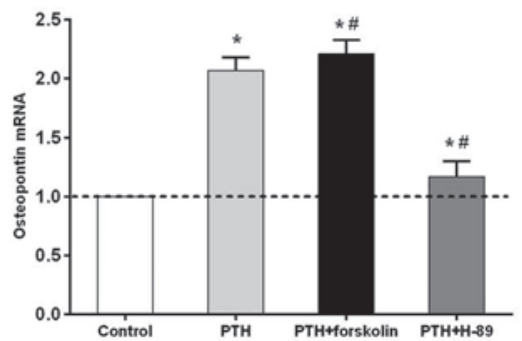

Figure 3. Reverse transcription-quantitative polymerase chain reaction analysis of osteogenic gene expression following different treatments. The PTH and $\mathrm{PTH}+$ forskolin groups demonstrated increased (A) RUNX2, (B) osterix, (C) collagen I, (D) osteocalcin and (E) osteopontin mRNA expression levels compared with the control group. The mRNA expression levels of these factors when treated with forskolin and PTH (1-34) were significantly increased compared with those when treated with PTH (1-34) alone. Following administration of PTH (1-34) and H-89, the mRNA expression of these factors significantly decreased compared with that in the PTH group. Data are expressed as mean \pm standard deviation $\left(\mathrm{n}=3\right.$ repeats). ${ }^{*} \mathrm{P}<0.05$ vs. control group; ${ }^{*} \mathrm{P}<0.05$ vs. $\mathrm{PTH}$ group. RUNX2, runt related transcription factor 2; PTH (1-34), parathyroid hormone (1-34).

\section{A}

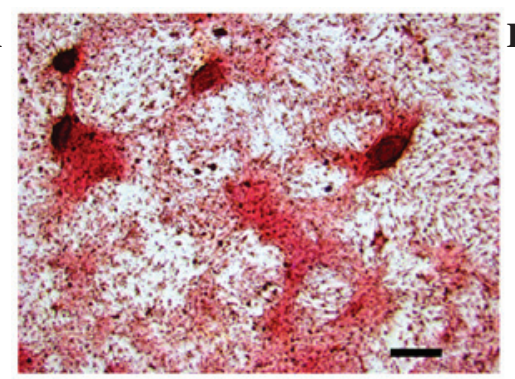

C

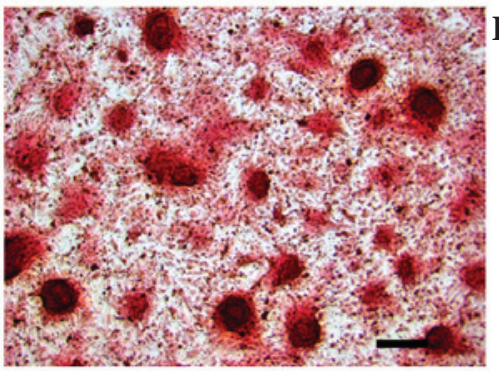

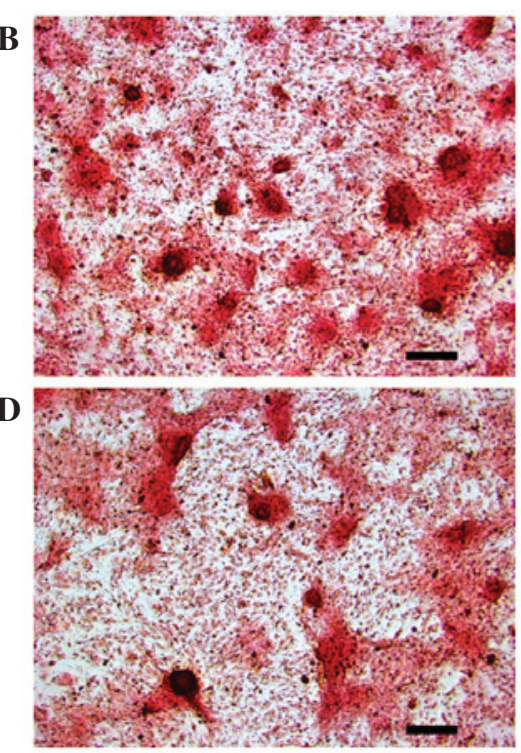

Figure 4. Intermittent PTH (1-34) treatment induced mineralization in a cyclic adenosine monophosphate/protein kinase A-dependent manner. Bone mesenchymal stromal cells in (A) control, (B) PTH, (C) PTH + forskolin and (D) PTH + H-89 groups were stained for calcium deposition using Alizarin Red S. The $\mathrm{PTH}$ and PTH + forskolin groups demonstrated increased mineralization compared with the control group. The mineralization effect in the PTH + forskolin group was more marked than that in the PTH group. However, PTH-induced mineralization was inhibited by H-89. Scale bar=200 $\mu \mathrm{m}$, brightfield. PTH (1-34), parathyroid hormone (1-34).

\section{Discussion}

Previous studies have demonstrated the anabolic effect of intermittent PTH (1-34) on bone formation $(23,24)$. Furthermore, a number of studies have suggested that $\mathrm{cAMP} / \mathrm{PKA}$ signaling is activated by PTH (1-34) during osteogenic differentiation $(8,9,21)$. However, precisely how the intermittent administration of PTH (1-34) regulates osteogenic differentiation of BMSCs via the cAMP/PKA signaling pathway requires elucidation. In the present study, the adenylyl cyclase activator forskolin and the specific PKA inhibitor H-89 were used. A cell proliferation assay, osteogenic gene testing, ALP activity detection, Alizarin Red S staining and detection of p-CREB, RUNX2 and osterix protein expression were used in the current study in order to determine the underlying molecular mechanism by which intermittent PTH (1-34) administration modulates the proliferation and osteogenic differentiation of BMSCs via the cAMP/PKA signaling pathway. The present 

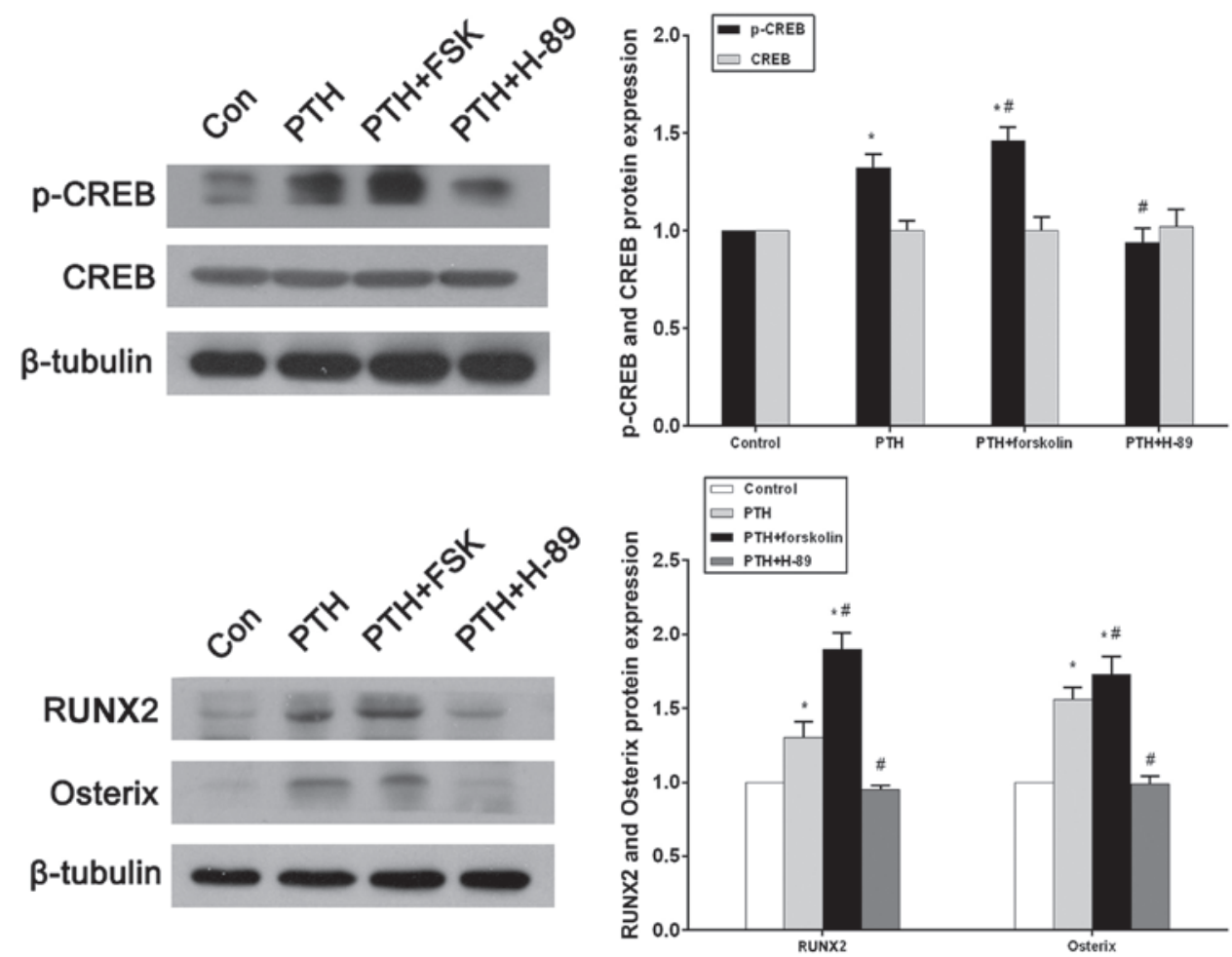

Figure 5. Effect of the cyclic adenosine monophosphate/protein kinase A signaling pathway on PTH (1-34)-induced osteogenic differentiation of bone mesenchymal stromal cells using western blot and densitometric analysis. Protein expression of p-CREB, RUNX2 and osterix in the PTH group were significantly increased compared with those in the control group, indicative of osteogenic differentiation. Addition of forskolin to PTH-induced cells resulted in an additional increase in p-CREB, RUNX2 and osterix protein expression, which was inhibited by treatment with H-89. Data are expressed as mean \pm standard deviation ( $\mathrm{n}=3$ repeats). ${ }^{*} \mathrm{P}<0.05$ vs. control group; ${ }^{\mathrm{P}} \mathrm{P}<0.05$ vs. $\mathrm{PTH}$ group. Con, control; $\mathrm{p}$-CREB, phosphorylated cAMP response element binding protein; PTH (1-34), parathyroid hormone (1-34); RUNX2, runt related transcription factor 2.

findings indicated that intermittent PTH (1-34) treatment regulates p-CREB to promote the proliferation and osteogenic differentiation of BMSCs through the cAMP/PKA pathway.

The results of the cell proliferation assay demonstrated that PTH (1-34) stimulated BMSC proliferation, forskolin enhanced PTH-stimulated BMSC proliferation and the stimulating effect of PTH (1-34) was blocked by H-89. The results indicated that intermittent PTH (1-34) treatment promoted proliferation of BMSCs through the cAMP/PKA pathway. Previously, Baron and Hesse (25) had also suggested that PTH stimulated the proliferation of MSCs. Nishida et al (26) cultured bone marrow cells isolated from the femora and tibiae of rats and treated them with PTH, reporting that PTH induced apparent increases in the total number of colony forming unit-fibroblasts. The findings of the present study add to the current body of knowledge about the effects of PTH (1-34) on BMSC proliferation via the cAMP/PKA pathway.

RUNX2 and osterix are transcription factors used in osteogenic lineage commitment expressed specifically at high levels in osteoblasts. Thus, RUNX2 and osterix may be considered to be osteoblast-specific transcription factors $(27,28)$. In support of the current results, Krishnan et al (29) revealed that PTH rapidly increases RUNX2 mRNA and protein levels through activation of the cAMP/PKA pathway and Wang et al (8) demonstrated that PTH stimulates RUNX2 and osterix mRNA expression via cAMP/PKA signaling. Furthermore, ALP is the most widely recognized marker of osteoblast phenotypes and has an important role during bone mineralization (30). A previous study reported that PTH promotes osteogenic differentiation from MSCs by enhancing ALP activity (7). Kao et al (16) reported that treating BMSCs with PTH increased the number of ALP-positive cells, and that activation of cAMP signaling in BMSCs with forskolin markedly enhanced ALP activity. It is also of note that collagen I is a focal component of the extracellular matrix in the bone, and the expression of collagen I typically occurs at the stage of matrix synthesis during osteogenesis (31-33). In addition, expression of osteocalcin and osteopontin mark the onset of mineralization (34). Kao et al (16) reported that direct activation of cAMP signaling by forskolin in BMSCs elevated the expression of osteoblast marker genes, such as RUNX2, osterix, collagen I and osteocalcin, and that treatment of BMSCs with PTH enhanced the ability of the subsequent differentiated osteoblasts to mineralize. As with PTH, exposing BMSCs to forskolin also increased mineralization. In the present study, and consistent with these previous studies, the results of the ALP activity assay and RT-qPCR revealed that ALP activity and RUNX2, osterix, collagen I, osteocalcin and osteopontin expression were all upregulated by intermittent PTH (1-34) application, and that the addition of forskolin enhanced these effects to a greater degree than was achieved by PTH (1-34) alone. Furthermore, the effects of PTH (1-34) were inhibited by H-89. Alizarin Red S staining revealed that intermittent PTH (1-34) treatment significantly increased mineralization via the cAMP/PKA pathway. Together, these data imply that intermittent PTH (1-34) administration can affect osteogenesis by increasing the commitment of BMSCs to the osteogenic lineage, via activation of the cAMP/PKA pathway. 
CREB is a cellular transcription factor (35) that is phosphorylated subsequent to cAMP activation of PKA. CREB then translocates into the nucleus, where it activates transcription of target genes (17). Siddappa et al (15) suggested that addition of dibutyryladenosine-cAMP to human MSCs for $6 \mathrm{~h}$ resulted in increased phosphorylation of the transcription factor CREB, which could be inhibited by treatment with H-89. Furthermore, Tyson et al (36) cultured UMR 106 cells and treated them with PTH, finding that PKA is the enzyme responsible for phosphorylating CREB in response to PTH. In the current study, the western blot analysis demonstrated that intermittent PTH (1-34) application upregulated the expression of $\mathrm{p}-\mathrm{CREB}$, that administration of forskolin significantly increased $\mathrm{p}$-CREB expression in addition to that observed with PTH treatment alone and that the effect of PTH on p-CREB expression could be blocked by H-89. This trend was also observed in the protein expression of RUNX2 and osterix.

In conclusion, the present in vitro study demonstrated that intermittent PTH (1-34) administration regulates the proteins downstream of the cAMP/PKA signaling pathway, such as $\mathrm{p}-\mathrm{CREB}$, to enhance the proliferation, osteogenic differentiation and mineralization of BMSCs.

\section{Acknowledgements}

This study was supported by the Guangdong Nature Science Foundation in 2013 (grant no. S2013010015784).

\section{References}

1. Pettway GJ, Meganck JA, Koh AJ, Keller ET, Goldstein SA and McCauley LK: Parathyroid hormone mediates bone growth through the regulation of osteoblast proliferation and differentiation. Bone 42: 806-818, 2008

2. Compston JE: Skeletal actions of intermittent parathyroid hormone: Effects on bone remodelling and structure. Bone 40: 1447-1452, 2007.

3. Neer RM, Arnaud CD, Zanchetta JR, Prince R, Gaich GA, Reginster JY, Hodsman AB, Eriksen EF, Ish-Shalom S, Genant HK, et al: Effect of parathyroid hormone (1-34) on fractures and bone mineral density in postmenopausal women with osteoporosis. N Engl J Med 344: 1434-1441, 2001.

4. Jiang Y, Zhao J, Liao EY, Dai RC, Wu XP and Genant HK: Application of micro-CT assessment of 3-D bone microstructure in preclinical and clinical studies. J Bone Miner Metab 23 (Suppl): S122-S131, 2005

5. Jilka RL, Weinstein RS, Bellido T, Roberson P, Parfitt AM and Manolagas SC: Increased bone formation by prevention of osteoblast apoptosis with parathyroid hormone. J Clin Invest 104: 439-446, 1999.

6. Kaback LA, Soung do Y, Naik A, Geneau G, Schwarz EM, Rosier RN, O'Keefe RJ and Drissi H: Teriparatide (1-34 human $\mathrm{PTH})$ regulation of osterix during fracture repair. J Cell Biochem 105: 219-226, 2008.

7. Yang C, Frei H, Burt HM and Rossi F: Effects of continuous and pulsatile PTH treatments on rat bone marrow stromal cells. Biochem Biophys Res Commun 380: 791-796, 2009.

8. Wang BL, Dai CL, Quan JX, Zhu ZF, Zheng F, Zhang HX, Guo SY, Guo G, Zhang JY and Qiu MC: Parathyroid hormone regulates osterix and Runx 2 mRNA expression predominantly through protein kinase A signaling in osteoblast-like cells. J Endocrinol Invest 29: 101-108, 2006.

9. Nakao Y, Koike T, Ohta Y, Manaka T, Imai Y and Takaoka K: Parathyroid hormone enhances bone morphogenetic protein activity by increasing intracellular 3',5'-cyclic adenosine monophosphate accumulation in osteoblastic MC3T3-E1 cells. Bone 44: 872-877, 2009.

10. Kulkarni NH, Halladay DL, Miles RR, Gilbert LM, Frolik CA, Galvin RJ, Martin TJ, Gillespie MT and Onyia JE: Effects of parathyroid hormone on Wnt signaling pathway in bone. J Cell Biochem 95: 1178-1190, 2005.
11. Inoue Y, Canaff L, Hendy GN, Hisa I, Sugimoto T, Chihara K and Kaji H: Role of Smad3, acting independently of transforming growth factor-beta, in the early induction of Wnt-beta-catenin signaling by parathyroid hormone in mouse osteoblastic cells. J Cell Biochem 108: 285-294, 2009.

12. Tian Y, Xu Y, Fu Q and He M: Parathyroid hormone regulates osteoblast differentiation in a Wnt/ $\beta$-catenin-dependent manner. Mol Cell Biochem 355: 211-216, 2011.

13. Miao D, Tong XK, Chan GK, Panda D, McPherson PS and Goltzman D: Parathyroid hormone-related peptide stimulates osteogenic cell proliferation through protein kinase $\mathrm{C}$ activation of the Ras/mitogen-activated protein kinase signaling pathway. J Biol Chem 276: 32204-32213, 2001.

14. Rey A, Manen D, Rizzoli R, Ferrari SL and Caverzasio J: Evidences for a role of p38 MAP kinase in the stimulation of alkaline phosphatase and matrix mineralization induced by parathyroid hormone in osteoblastic cells. Bone 41: 59-67, 2007.

15. Siddappa R, Martens A, Doorn J, Leusink A, Olivo C, Licht R, van Rijn L, Gaspar C, Fodde R, Janssen F, et al: cAMP/PKA pathway activation in human mesenchymal stem cells in vitro results in robust bone formation in vivo. Proc Natl Acad Sci USA 105: 7281-7286, 2008.

16. Kao R, Lu W, Louie A and Nissenson R: Cyclic AMP signaling in bone marrow stromal cells has reciprocal effects on the ability of mesenchymal stem cells to differentiate into mature osteoblasts versus mature adipocytes. Endocrine 42: 622-636, 2012.

17. Doorn J, Siddappa R, van Blitterswijk CA and de Boer J: Forskolin enhances in vivo bone formation by human mesenchymal stromal cells. Tissue Eng Part A 18: 558-567, 2012.

18. Yang DC, Tsay HJ, Lin SY, Chiou SH, Li MJ, Chang TJ and Hung SC: cAMP/PKA regulates osteogenesis, adipogenesis and ratio of RANKL/OPG mRNA expression in mesenchymal stem cells by suppressing leptin. PLoS One 3: e1540, 2008.

19. Potts JT: Parathyroid hormone: Past and present. J Endocrinol 187: 311-325, 2005.

20. Zimmermann M: Ethical guidelines for investigations of experimental pain in conscious animals. Pain 16: 109-110, 1983.

21. Kawane T, Mimura J, Yanagawa T, Fujii-Kuriyama Y and Horiuchi N: Parathyroid hormone (PTH) down-regulates $\mathrm{PTH} / \mathrm{PTH}$-related protein receptor gene expression in UMR-106 osteoblast-like cells via a 3',5'-cyclic adenosine monophosphate-dependent, protein kinase A-independent pathway. J Endocrinol 178: 247-256, 2003.

22. Livak KJ and Schmittgen TD: Analysis of relative gene expression data using real-time quantitative PCR and the 2(-Delta Delta C(T)) Method. Methods 25: 402-408, 2001.

23. Nozaka K, Miyakoshi N, Kasukawa Y, Maekawa S, Noguchi H and Shimada Y: Intermittent administration of human parathyroid hormone enhances bone formation and union at the site of cancellous bone osteotomy in normal and ovariectomized rats. Bone 42: 90-97, 2008.

24. Rickard DJ, Wang FL, Rodriguez-Rojas AM, Wu Z, Trice WJ, Hoffman SJ, Votta B, Stroup GB, Kumar S and Nuttall ME: Intermittent treatment with parathyroid hormone (PTH) as well as a non-peptide small molecule agonist of the PTH1 receptor inhibits adipocyte differentiation in human bone marrow stromal cells. Bone 39: 1361-1372, 2006.

25. Baron R and Hesse E: Update on bone anabolics in osteoporosis treatment: Rationale, current status, and perspectives. J Clin Endocrinol Metab 97: 311-325, 2012.

26. Nishida S, Yamaguchi A, Tanizawa T, Endo N, Mashiba T, Uchiyama Y, Suda T, Yoshiki S and Takahashi HE: Increased bone formation by intermittent parathyroid hormone administration is due to the stimulation of proliferation and differentiation of osteoprogenitor cells in bone marrow. Bone 15: 717-723, 1994.

27. Nakashima K and de Crombrugghe B: Transcriptional mechanisms in osteoblast differentiation and bone formation. Trends Genet 19: 458-466, 2003.

28. Marie PJ: Transcription factors controlling osteoblastogenesis. Arch Biochem Biophys 473: 98-105, 2008.

29. Krishnan V, Moore TL, Ma YL, Helvering LM, Frolik CA, Valasek KM, Ducy P and Geiser AG: Parathyroid hormone bone anabolic action requires Cbfa1/Runx2-dependent signaling. Mol Endocrinol 17: 423-435, 2003.

30. Stucki U, Schmid J, Hämmerle CF and Lang NP: Temporal and local appearance of alkaline phosphatase activity in early stages of guided bone regeneration. A descriptive histochemical study in humans. Clin Oral Implants Res 12: 121-127, 2001. 
31. Franceschi RT: The developmental control of osteoblast-specific gene expression: Role of specific transcription factors and the extracellular matrix environment. Crit Rev Oral Biol Med 10: 40-57, 1999.

32. Zhou Y, Guan X, Zhu Z, Gao S, Zhang C, Li C, Zhou K, Hou W and Yu H: Osteogenic differentiation of bone marrow-derived mesenchymal stromal cells on bone-derived scaffolds: Effect of microvibration and role of ERK1/2 activation. Eur Cell Mater 22: $12-25,2011$.

33. Zhang C, Li J, Zhang L, Zhou Y, Hou W, Quan H, Li X, Chen Y and $\mathrm{Yu} \mathrm{H}$ : Effects of mechanical vibration on proliferation and osteogenic differentiation of human periodontal ligament stem cells. Arch Oral Biol 57: 1395-1407, 2012.

34. Owen TA, Aronow M, Shalhoub V, Barone LM, Wilming L, Tassinari MS, Kennedy MB, Pockwinse S, Lian JB and Stein GS: Progressive development of the rat osteoblast phenotype in vitro: Reciprocal relationships in expression of genes associated with osteoblast proliferation and differentiation during formation of the bone extracellular matrix. J Cell Physiol 143: 420-430, 1990.
35. Bourtchuladze R, Frenguelli B, Blendy J, Cioffi D, Schutz G and Silva AJ: Deficient long-term memory in mice with a targeted mutation of the cAMP-responsive element-binding protein. Cell 79: 59-68, 1994

36. Tyson DR, Swarthout JT and Partridge NC: Increased osteoblastic c-fos expression by parathyroid hormone requires protein kinase A phosphorylation of the cyclic adenosine 3',5'-monophosphate response element-binding protein at serine 133 Endocrinology 140: 1255-1261, 1999. 